\title{
ANÁlISE QUALITATIVA DA ARBORIZAÇÃO DA AV. PEDRO DUAILIBE EM BARREIRAS DO PIAUÍ-PI
}

\author{
MARLOS A. DE SENA-IFPI ${ }^{1}$, \\ GRAZIELLE N. DE OLIVEIRA-IFPI ${ }^{1}$, \\ ROSANGELA BATISTA-IFPI ${ }^{1}$, \\ SAVANNA A. DA SILVA-IFPI ${ }^{1}$, \\ WANES R. ROCHA-IFPI ${ }^{1}$, \\ BRUNA DE FREITAS IWATA - IFPI.
}

\begin{abstract}
RESUMO: A realização de análise qualitativas das árvores plantadas no ambiente urbano é essencial para manutenção da qualidade da vida urbana pois permite identificar os principais problemas e propor possíveis soluções. Dentre desse contexto, este trabalho foi realizado com o objetivo de avaliar os impactos negativos social e econômico da arborização de uma das principais avenidas pública de Barreiras do Piauí-PI. A análise foi realizada por meio de censo, no qual os indivíduos foram quantificados e caracterizados de acordo com o seguinte parâmetros: Interferências geradas pela relação arvore-construção/fiação. Foi observado também se os indivíduos sofreram algum tipo de manejo e, posteriormente, sugeridas quais as ações seriam necessárias para a sua adequação. Foram avaliados no total 48 indivíduos, distribuídos em variáveis tipos de espécies. A maioria dos indivíduos apresentou irregularidades.
\end{abstract}

Palavras-Chave: Monitoramento Arbóreo, Planejamento Urbanístico, Qualidade Ambiental.

ABSTRACT: The realization of qualitative analysis of trees planted in the urban environment is essential for maintaining the quality of urban life as it allows to identify the main problems and propose possible solutions. One of this context, this study was conducted to assess the negative social and economic impacts of afforestation of one of the main avenues of public Barriers do Piauí-PI. The analysis was performed by means of census, in which individuals were quantified and characterized according to the following parameters: Interference generated by treeconstruction/wiring. Noted individuals also have suffered some kind of management and, subsequently, suggested what actions would be needed for its suitability. A total of 48 individuals were evaluated, distributed in types of species. The majority of individuals presented irregularities.

Keywords: Tree Monitoring, Urban Planning, Environmental Quality. 


\section{INTRODUÇÃO}

A existência da arborização urbana é essencial para o conforto humano e ao meio ambiente quando planejada corretamente. Os conflitos gerados pela implantação da arborização inadequada podem ser observados nas interferências e prejuízos causados aos equipamentos e estruturas urbanas, tais como: fiações elétricas, encanamentos, calhas, calçamentos, muros, postes de iluminação e sinalização. Estes problemas fáceis visualizados contribuem para a deficiência de uma cidade consequentemente gerando conflitos para a sociedade, em termos sociais e econômicos, (Buckeridge, 2015).

Considerando esses aspectos, a análise qualitativa das árvores urbanas desempenha um papel fundamental no planejamento da arborização no momento em que permite prever o surgimento desses conflitos, identificar os atuais e propor soluções adequadas ás características do local avaliado, para que possa ambas as partes sociais e ambientais sejam beneficiadas ecologicamente, (Santos et al, 2015).

A falta de planejamento da arborização permite que iniciativas particulares, pontuais e desprovidas de conhecimento técnico adequado, ocupem os espaços com plantio irregulares de árvores em locais sem enquadramento com o planejamento posterior de ruas e avenidas de uma cidade. Esse procedimento compromete com o espaçamento adequado das mesmas, causando conflito com o trafego de veículos e fiação elétrica, causando muitas vezes sérios prejuízos e transtornos, (Santos et al, 2015).

Depois de pensados todos os aspectos que envolvem o planejamento da arborização urbana, pois não seria útil ter-se um projeto bem elaborado e bem executado se não for continuado. No caso de projeto de arborização, algumas atividades de manejo são comumente necessárias para a sobrevivência e o bom desenvolvimento das árvores urbanas, lembrando que se a implantação for bem planejada as manutenções necessárias serão diminuídas.

O inventario das arvores é uma ferramenta para as etapas de manejo, e no caso específico da arborização urbana, busca-se obter informações quali-quantitativas que possibilitam conhecer o patrimônio arbóreo e identificar as atividades de manejo mais necessárias.

Diante desse contexto, este trabalho foi realizado com o objetivo de avaliar qualitativamente a arborização urbana de uma das principais avenidas públicas de Barreiras do 
Piauí - PI e identificar os principais problemas e as principais necessidades para que se possa planejar um manejo adequado.

\section{MATERIAL E MÉTODOS}

\section{Área de Estudos}

O presente trabalho foi realizado no município de Barreiras do Piauí-PI, localizado na região sul do estado, promoveu sua elevação a sede municipal, em 1962 (9 $9^{\circ}$ 54'6" SU E 45 28`37' Oeste). Possui uma população de 3.234 habitantes (IBGE, resultado censo 2010). A área estudada foi a Avenida Pedro Duailibe, na qual a análise foi realizada por meio de um censo em uma das principais avenidas da cidade, no período de fevereiro de 2016, com finalidades de apontar fatos negativos em uma projeção de arborização urbana.

\section{Procedimentos}

O levantamento das informações foi através de estudos in loco, com o levantamento das especificações dos indivíduos foi dada a partir de $1 \mathrm{~m}$ de CAP (circunferência a altura do peito). O estudo foi baseado no inventário quali-quantitativo de todos os indivíduos presente na avenida. O estudo levantou as seguintes variáveis: Altura total, CAP (circunferência a altura do peito), DAP (diâmetro a altura do peito), origem, nome popular, espécie, sombreamento, metragem da avenida $\mathrm{em}^{2}$ intervalo de espaçamento dos indivíduos. Sendo usado: Trena métrica de $50 \mathrm{~m} \mathrm{e}$ um suporte da madeira, para obtermos determinados dados os dados quantitativos.

\section{RESULTADOS E DISCUSSÕES}

O estudo verificou a ocorrência de 48 indivíduos, pertencentes a 12 espécies, sendo que 8 (nove) espécies não foram Identificadas (Tabela 1). Relatando a predominante da espécie Oiti. Fato relacionado com estudos das 25 vias públicas da Cidade Aracaju - SE, (Santos et al, 2015), sendo predominante ao mesmo tipo de espécie.

Tabela 1. Inventario de indivíduos arbóreos presentes na Avenida Pedro Duailibe, Barreiras do Piaui.

\begin{tabular}{llll}
\hline NOMES CIENTÍFICOS & NOMEPOPU & NI & ORIGEM \\
& LAR & & \\
\hline TAMARINDUS INDICAL & tamarindo & 01 & exótica \\
COPERNICLA PRUNIFERA & carnaúba & 05 & nativa
\end{tabular}




\begin{tabular}{llll} 
LICONLA TOMENTOSA & oiti & 18 & nativa \\
PACHIRA AQUATICA & munguba & 04 & nativa \\
CASALPINA ENCHINATA & Pau-brasil & 01 & nativa \\
GENIPA AMERICANA & jenipapo & 01 & nativa \\
MANGIFER INDICA & manga & 03 & exótica \\
ZIZIPOS JOAZEIRO & juá & 01 & nativa \\
AMYGDALUS COMUNIS & amêndoa & 03 & exótica \\
DYPISIS LUTES CENS & palmeira-areca & 01 & exótica \\
ANNONA SQUAMOSAL & ata & 01 & nativa \\
DILTERYX ALATA & baru & 01 & nativa \\
NÃO IDENTIFICADA & - & 08 & - \\
TOTAL & 12 & 48 & - \\
\hline
\end{tabular}

Fonte: Levantamento de campo, 2016.

No levantamento de dados referente as medidas do CAP, DAP, atura total, atura da copa e do fuste, obtemos dados como mostra figura abaixo (Tabela 2), Estude que nos permite sabermos as dimensões de cada indivíduo arbóreo, percebendo que a uma predominância de todos os dados da espécie oiti.

Tabela 2, resultados dos DAP, CAP, fuste, altura total, altura da copa, em média de espécies, dos individuo arbóreos da Av. Pedro Duilibe, Barreiras-PI.

\begin{tabular}{lllllll}
\hline NOMES CIENTÍFICOS & NOME & H.T & H.F & CAP & DAP & H.C \\
& POPULAR & & & & & \\
\hline TAMARINDUS INDICAL & tamarindo & 9 & 4,62 & 2,51 & 0,47 & 4,38 \\
COPERNICLA PRUNIFERA & carnaúba & 26,16 & 8,1 & 4,32 & 1,37 & 10,32 \\
LICONIA TOMENTOSA & oiti & 133,8 & 73,37 & 47,41 & 10,74 & 60,34 \\
PACHIRA AQUATICA & munguba & 40 & 18,82 & 6,38 & 2,93 & 2,64 \\
CASALPINA ENCHINATA & Pau-brasil & 4,18 & 2,67 & 0,18 & 0,05 & 0,51 \\
GENIPA AMERICANA & jenipapo & 5,30 & 3,63 & 1,10 & 0,35 & 1,67 \\
MANGIFER INDICA & manga & 3,45 & 9,05 & 3,97 & 1,25 & 14,4 \\
ZIZIPOS JOAZEIRO & juá & 8,30 & 7,85 & 1 & 3,31 & 0,45 \\
AMYGDALUS COMUNIS & amêndoa & 6,78 & 8,07 & 4,09 & 1,28 & 8,71 \\
DYPISIS LUTES CENS & palmeira-areca & 2,45 & 1,38 & 0,24 & 0,07 & 1,07 \\
ANNON A SQUAMOSAL & ata & 2,30 & 1,30 & 0,47 & 0,13 & 1,0
\end{tabular}




$\begin{array}{lllllll}\text { DILTERYX ALATA } & \text { barn } & 8,75 & 1,90 & 1,30 & 0,41 & 0,45 \\ \text { NÄO IDENTIFICADA } & - & 55,95 & 27,88 & 8,38 & 3,95 & 28,05\end{array}$

Fonte: Levantamento de campo, 2016.

Foi percebido que nesta via pública indivíduos arbóreos invadiam as guias de rolamento, interferindo o trafego de pessoas e veiculo, gerando desconforto social desta sociedade (Tabela 3).

Tabela 3. Indivíduos arbóreos em conflito direto com o transito pela guia de rolamento, Av. Pedro Duailibe.

\begin{tabular}{llll}
\hline Nomes Cientificos & N. Populares & Quantidades & Origem \\
\hline LICANIA TOMENTOSA & oiti & 02 & nativa \\
COPERNICLA PRUNIFERA & carnaúba & 01 & nativa \\
PACHIRA AQUATICA & munguba & 01 & nativa \\
N $\tilde{A O}$ IDENTIFICADA & - & 01 & - \\
\hline
\end{tabular}

Fonte: Levantamento de campo, 2016.

Notamos que em planejamento de arborização urbana, devemos cumprir todos os requisitos abordado em projeto, para que não ocorra erros que possa gerar conflitos sociais e econômico, com mostra a Figura 1 (A, B, C e D).

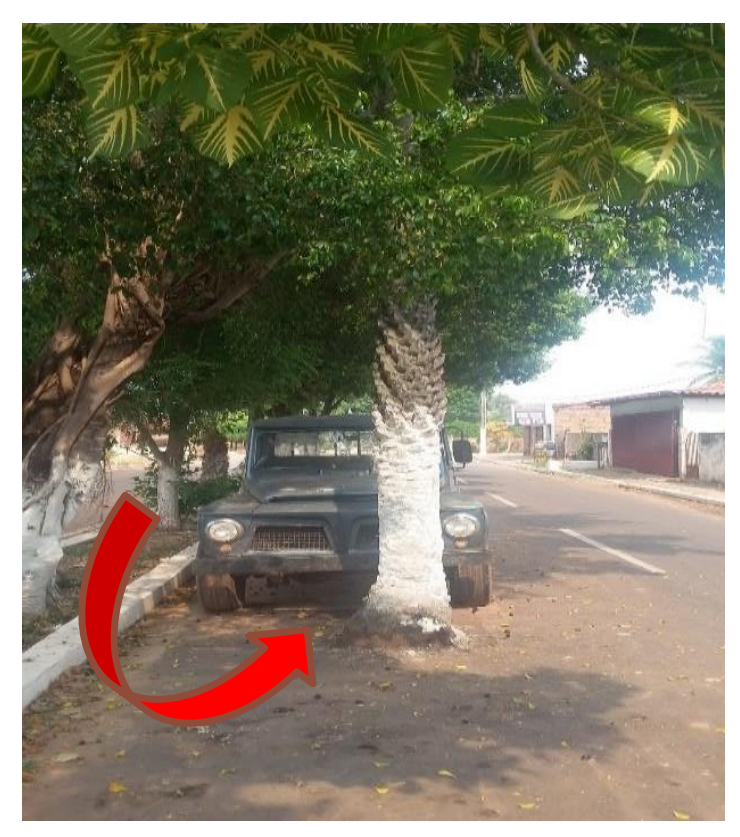

B)

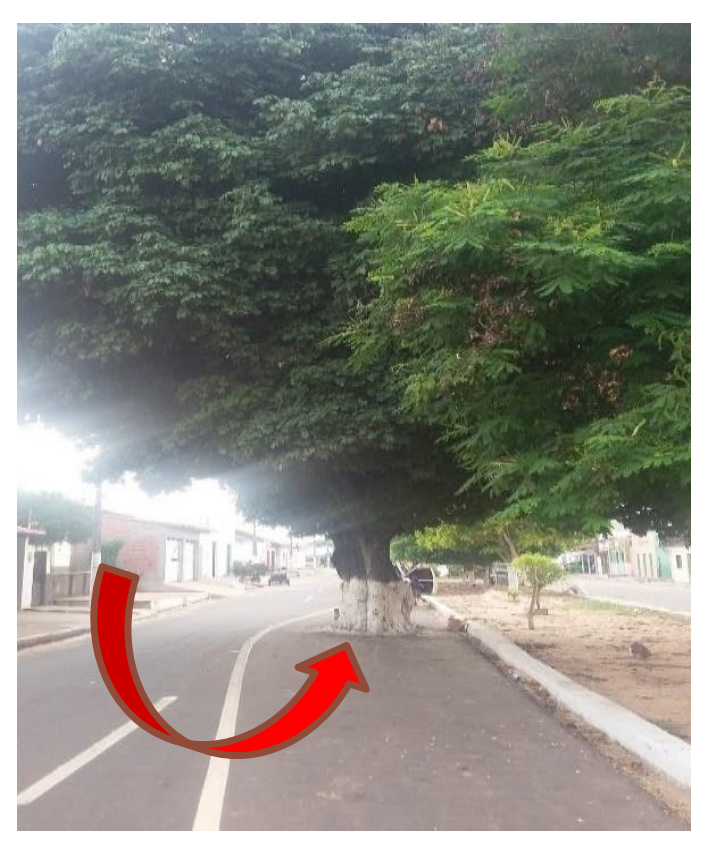




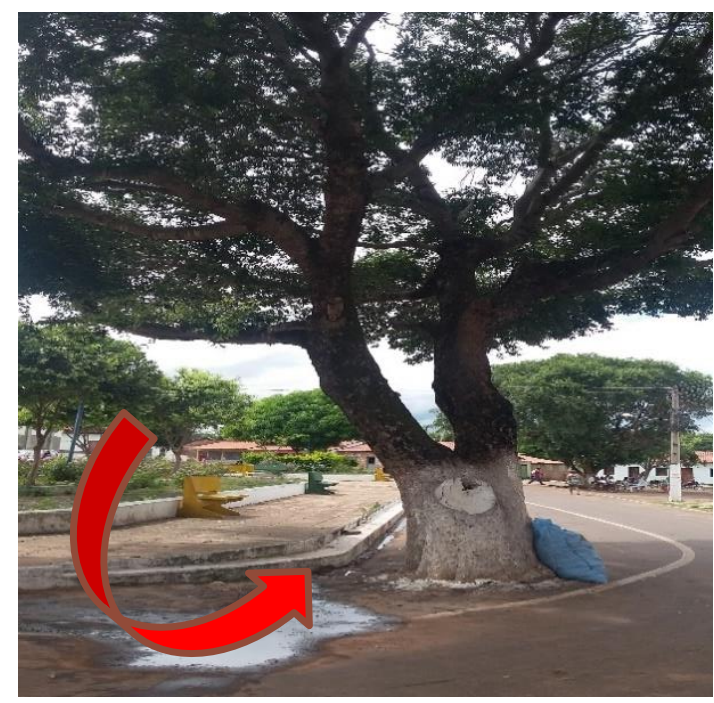

C)

D)

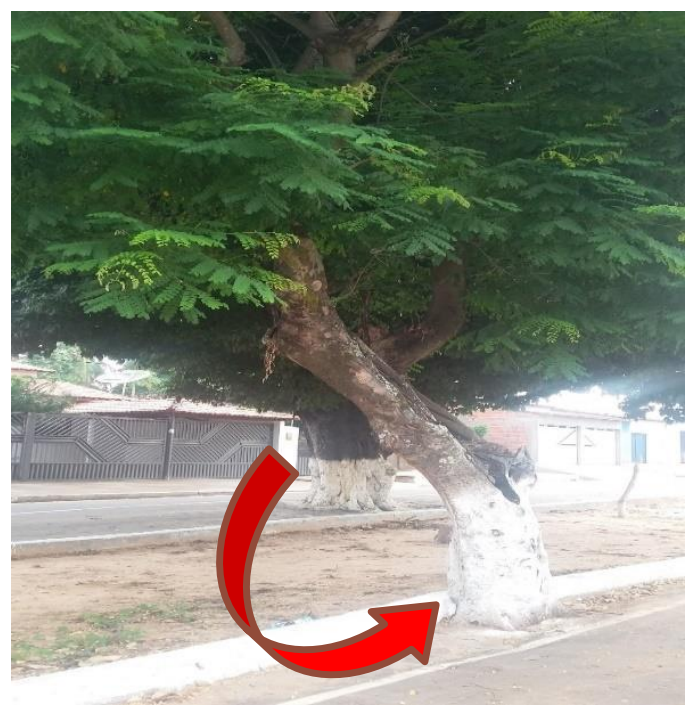

Figura 1. Indivíduos arbóreos de grande porte plantados no espaço da guia de rolamento da Avenida Pedro Duailibe, Barreiras do Piauí, PI. Fonte: Levantamento de campo, 2016.

Imagens de indivíduos arbóreos, relação direta com as guias de rolamento da Av. Pedro Duailibe, em Barreiras do Piaú-PI. Dificultando o trafego significativamente como mostra, (croqui, figura A).

Figura:A

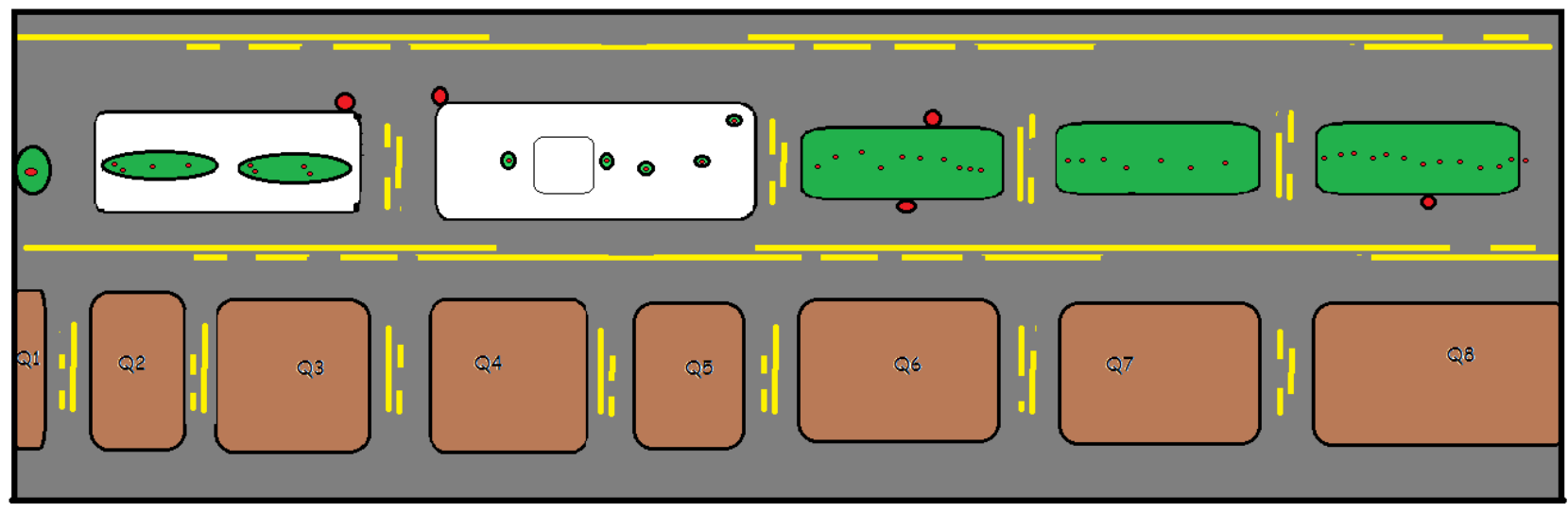

Avenida Pedro Duailibe, Barreiras do Piauí-PI.

Fonte: Levantamento de campo, 2016.

Q Quadras de rune

Dentre os dados obtidos em altura total dos indivíduos, podemos perceber que a maioria possui grande porte, vindo a ocasionar vários conflitos com fiação elétrica desprotegida. No entanto não sendo somente uma preocupação social, quanto econômica, tendo manutenção com maior frequência (Tabela 4).

Tabela 4. Conflitos arbóreos com a fiação elétrica da Av. Pedro Duailibe, Barreiras do Piaui-PI. 


\begin{tabular}{llll}
\hline Nomes Cientificos & N. Populares & Quantidade & Origem \\
\hline LINCONLA TOMENTOSA & Oiti & 05 & nativa \\
PACHIRA AQUATICA & munguba & 01 & nativa \\
\hline
\end{tabular}

Fonte: Levantamento de campo, 2016.

Em planejamento urbano devemos considerar a medida apropriadas para cada indivíduo arbóreo em relação com o meio físico, como mostra figuras abaixo de indivíduos em conflito com fiação elétrica, e supostos que virão no futuro da Av. Pedro Duailibe, como mostra figura (A).

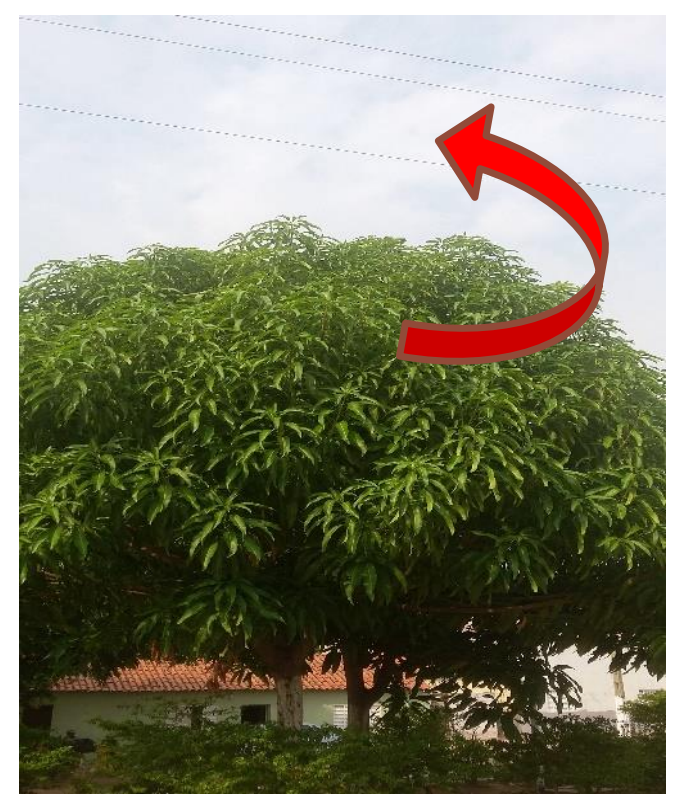

A)
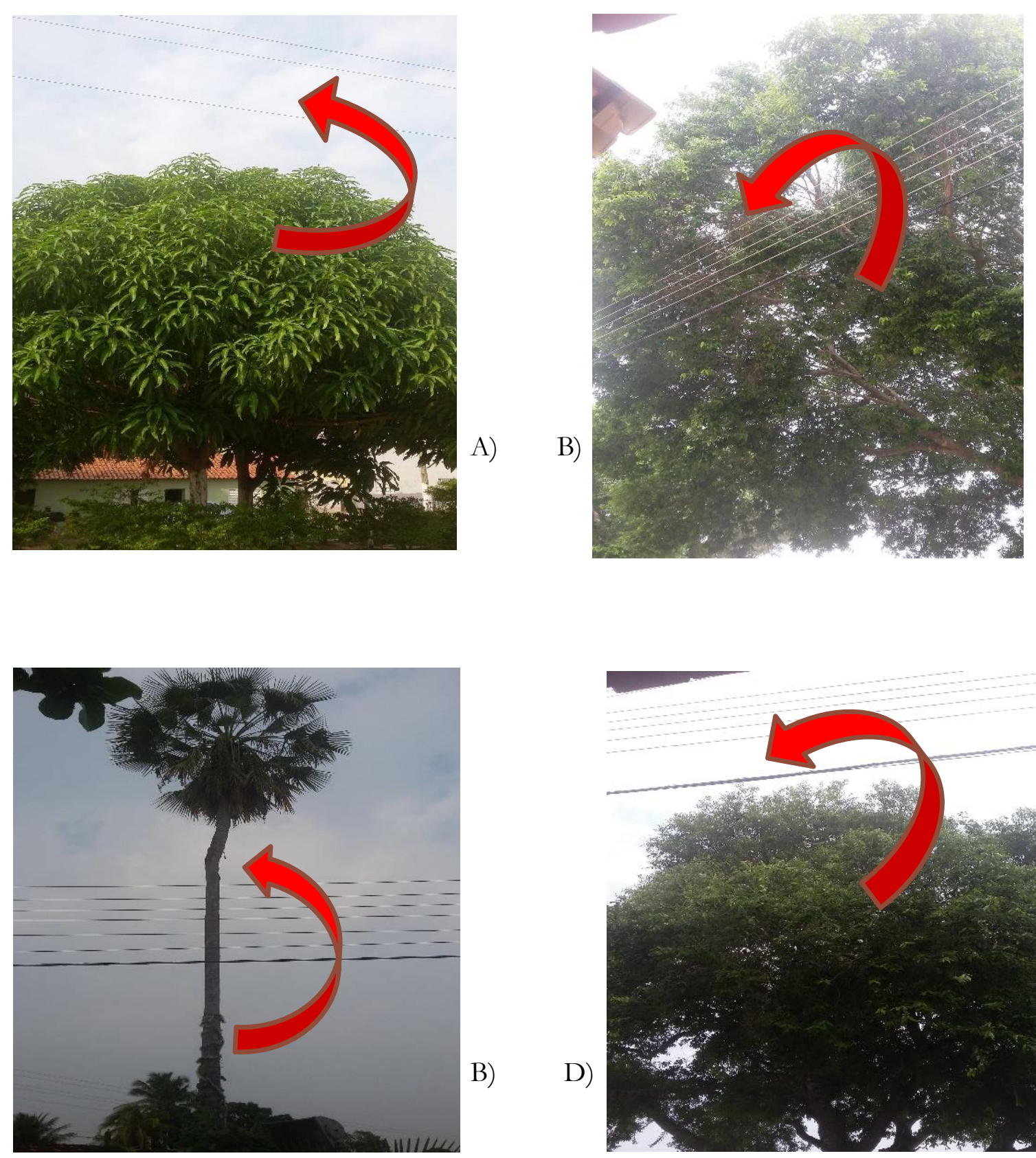

B)

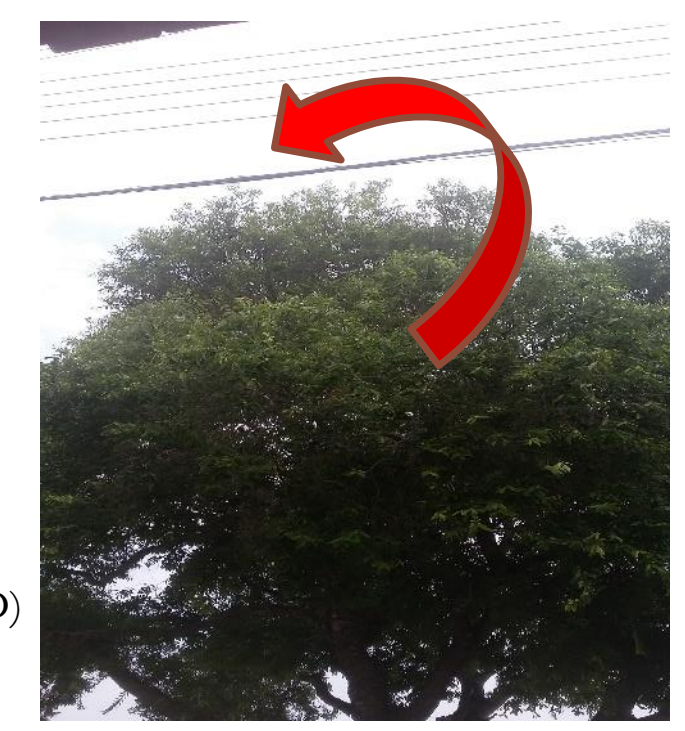


Figura 2. Indivíduos arbóreos em conflito com a fiação elétrica da Av. Pedro Duailibe, Barreiras do Piauí-PI. Fonte: Levantamento de campo, 2016

Outro problema bastante comum na arborização das cidades brasileiras é a implantação de espécies que apresentam sistema radicular superficial. Dessa forma, conhecer as espécies que apresentam essa característica, no planejamento da arborização urbana, constitui uma análise fundamental. Espécies que apresentam raízes com crescimento radicial superficial e pouco crescimento em profundidade, geralmente provocam problemas na arborização das vias, destruindo calçadas e canteiros, e, muitas vezes, comprometendo a estrutura de imóveis. A falta de espaço adequado nos canteiros para o desenvolvimento da árvore é uma das principais causas destes conflitos entre esse tipo de raiz e os elementos do meio (Santos e outros).

Tabela 5. Indivíduos arbóreos em conflito com pavimentação e calçamento

\begin{tabular}{llll}
\hline Nomes Cientificos & N. Populares & Quantidade & Origem \\
\hline LINCONLA TOMENTOSA & Oiti & 04 & nativa \\
$P A C H I R A$ AQUATICA & munguba & 01 & nativa \\
\hline
\end{tabular}

Fonte: Levantamento de campo, 2016.

Em relação a individuo arbóreo com raízes superficiais, nota-se que causam transtornos com a pavimentação de uma determinada cidade.

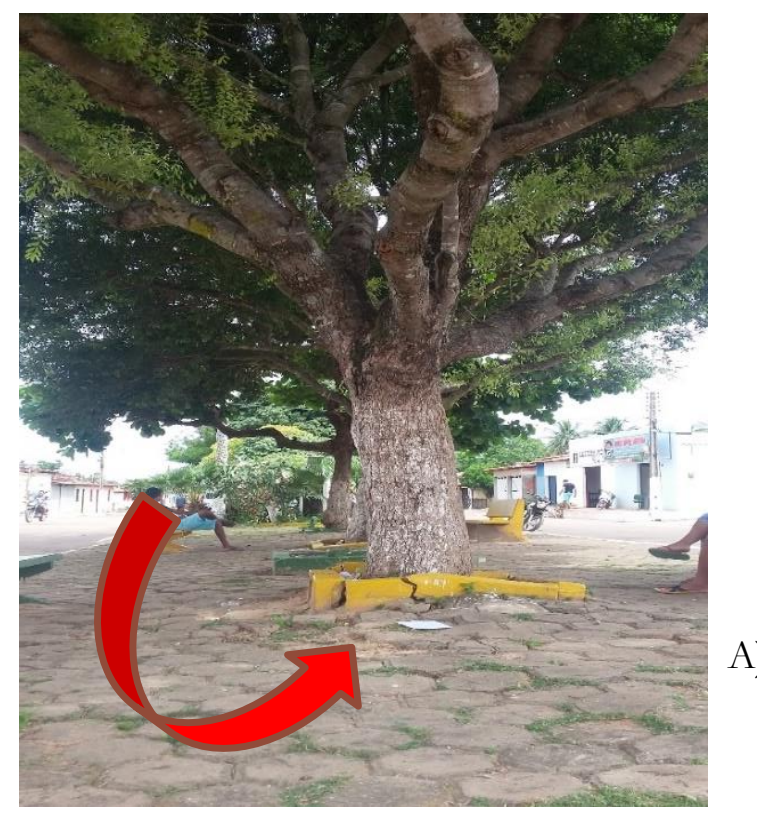

A)

B)

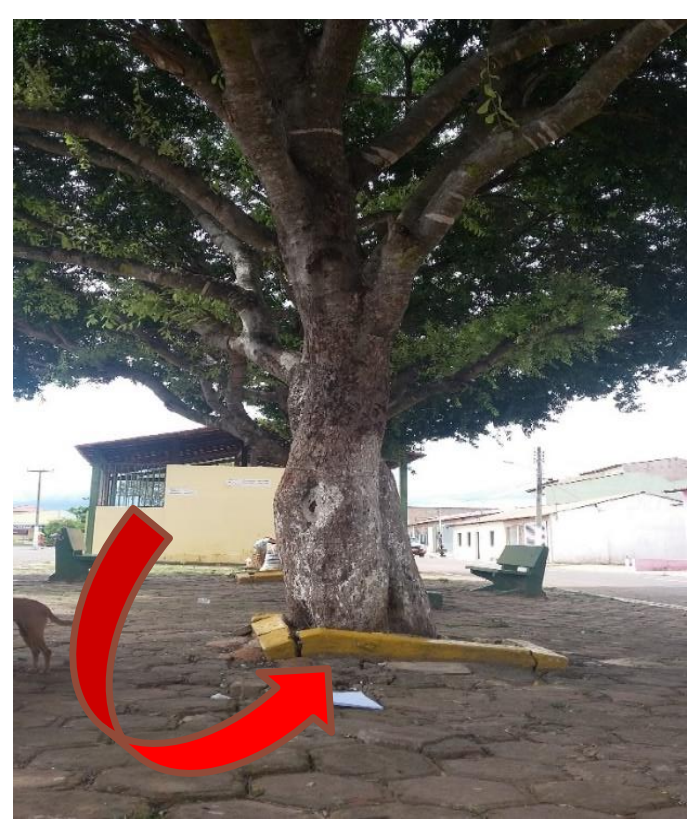



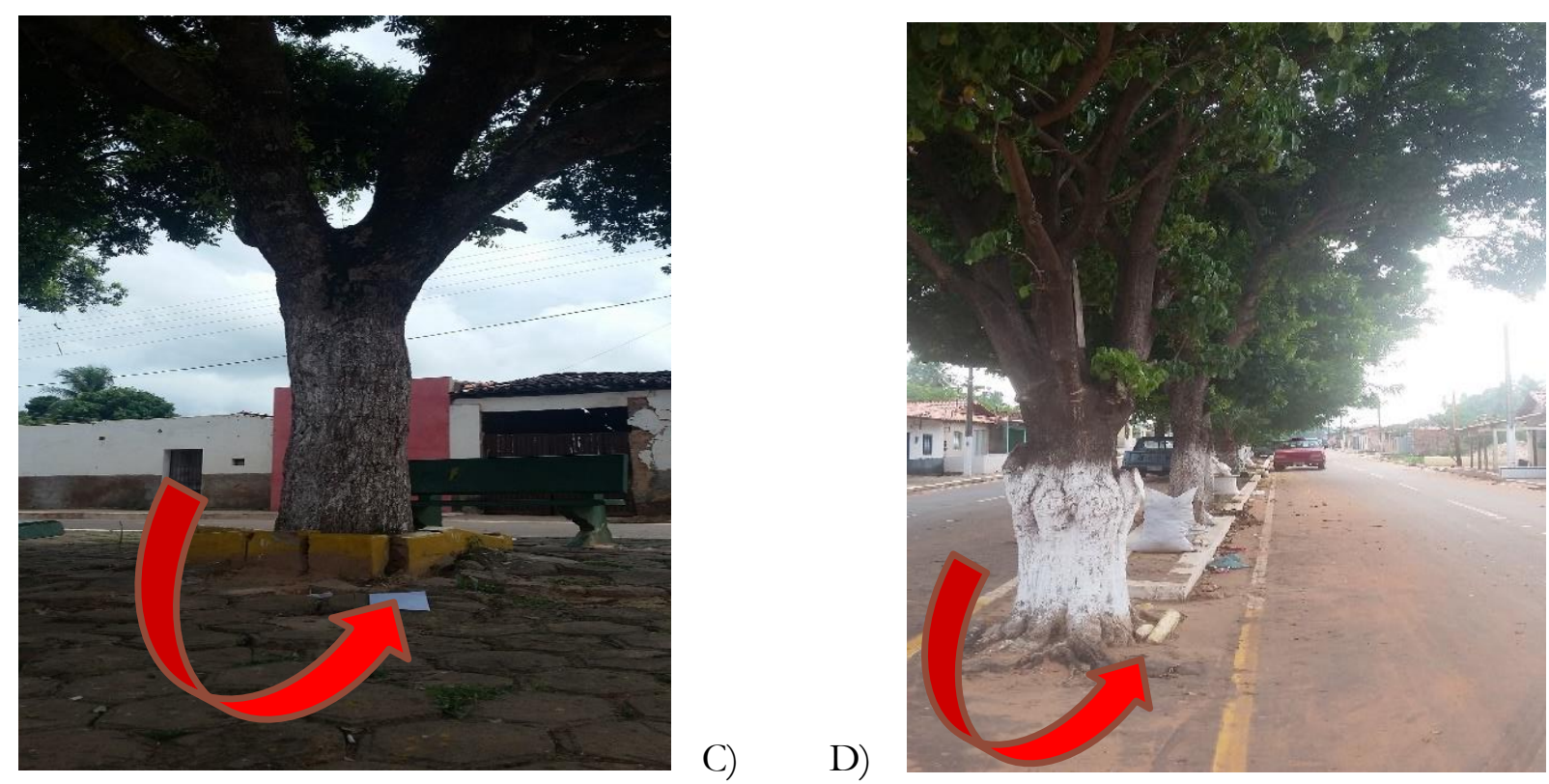

Fonte: Levantamento de campo, 2016.

Dados mostra que na Av. Pedro Duailibe, uma metragem de 7.124,8 $\mathrm{m}^{2}$ e sombreamento de $0,2556801594 \mathrm{~m}^{2}$ (Tabela 6).

Tabela 6. Área de sombreamento por presença de individuo arbóreo na Avenida Pedro Duailibe, Barreiras do Piauí, PI.

\begin{tabular}{ccc}
\hline Total de indivíduos & Área total de sombra & Sombreamento médio \\
\hline 49 & $1.821,67$ & 0,2556801594 \\
\hline
\end{tabular}

Fonte: Levantamento de campo, 2016.

\section{CONCLUSÃO}

Os principais conflitos sociais e econômicos vinculados a arborização da Av. Pedro Duilibe, Barreias do Piauí-PI foram: que os indivíduos arbóreos invadem as guias de rolamento estando em contato com a fiação elétrica e provocando degradação da pavimentação e calçamento deste Município. Neste decorrer notamos que de todos os dados obtidos houve uma predominância da espécie Oiti.

\section{REFERÊNCIAS BIBLIOGRÁFICAS}


Buckeridge. M. Árvores Urbanas em São Paulo: Planejamento, e Economia e Água. Estudo. Av. vol. 29 no. 84 São Paulo May/Avg. 2015.

Malos et al. Análise Qualitativa da Arborização da Av. Pedro Duailibe em Barreiras do Piauí-PI. 2016.

Santos et al. Análise Qualitativo da Arborização Urbana de 25 Vias Pública da Cidade de AracajuSE. Ciência. Flores. Vol. 25 no. 3 Santa Maria July/Sept. 2015. 